\title{
Research on Impedance Adaptive Correction Method for Low Voltage Short Circuit Test System
}

\author{
Yingmin You ${ }^{1,2}$, Wei Qiu², Xiangou Zhu' ${ }^{2}$, Jingqin Wang ${ }^{1, *}$ and Ziran $\mathrm{Wu}^{2}$ \\ ${ }^{1}$ State Key Laboratory for reliability and intelligence of electrical equipment, Hebei University of Technology, Tianjin, China \\ ${ }^{2}$ The Engineering Technology Research Center of Low-Voltage Apparatus of Zhejiang province, Wenzhou University, China \\ ${ }^{*}$ Corresponding author
}

\begin{abstract}
Before going public, low voltage circuit breaker must be tested, the rated operation short circuit breaking capacity and the rated limit short circuit breaking capacity test are particularly key. Therefore, the short circuit test device must be accurate and reliable, and the parameters which meet the customer requirements are set quickly and accurately. The traditional method needs to calculate the test parameters manually and manually check the table and it can not predict the system error impedance accurately. As a result, there is a large error in the numerical calculation. It is necessary to adjust several times to get accurate test parameters. This paper will introduce a method to quickly set test parameters, obtain accurate short circuit current, adjust the impedance of a short circuit system, and use this method to design a visual software with the functions of parameter calculation, acquisition of collected data and measurement, data query of impedance knife switch combination and self adaptation correction of impedance. By this method, the test parameters can be set up quickly, and the accuracy and efficiency can be improved.
\end{abstract}

Keywords—short circuit test; impedancecorrection; visualization software

\section{INTRODUCTION}

Low voltage electrical apparatus is widely used in various distribution electric fields, and it is essential for the verification of various properties of its products. Therefore, in order to improve the consistency and repeatability of the test results of relevant products, it is necessary to study the measurement and control technology of low voltage electrical apparatus. In the future, with the progress of computer hardware and software, the trend of low voltage electrical apparatus detection technology include few test times and high prediction precision ${ }^{[1-2]}$.

The short circuit test of low voltage circuit breaker is particularly important. The device that requires short circuit test must be accurate and reliable. For short circuit connection and breaking ability test, the test parameters need to be set quickly to obtain the demand of accurate short circuit current. This paper presents a visual software that can adjust the impedance of the short circuit system, it can calculate the impedance parameters, read the TDMS format data of the expected wave and query the switch data of the impedance knife and so on. In order to save a lot of time, the software can get more accurate short circuit impedance by considering the system impedance error and improve the accuracy. Because of the error in the system test, it is necessary to correct the impedance of the obtained data and then display it. In the obtained waveform, the value near zero is the DC component and the DC component is removed. The data obtained from the component is the corrected data ${ }^{[3-4]}$. And through the traditional method to obtain the parameters of short-circuit test steps, compared with the methods introduced in this paper, it can be seen that the steps of this method are highly efficient.

\section{LOW Voltage Circuit Breaker Short Circuit Test SYSTEM}

The circuit structure of the low voltage circuit breaker short-circuit experiment system is shown in Figure I. The 10KV signal is input to the impact test transformer through the switch cabinet. According to the requirements of the test parameters, the shock transformer is set by the star or triangle connection. Voltage modulation is used to meet the test requirements, and then input to the low voltage and the protection phase selection cabinet. The load (resistance and inductance load combination) is finally output to the test port. When conducting a small current test, the knife switch must be switched from the ground position to the back stage load, and then the grounding is carried out, as shown in Figure II. In addition, the system will connect the data acquisition device to the test port, which can collect test data in real time.

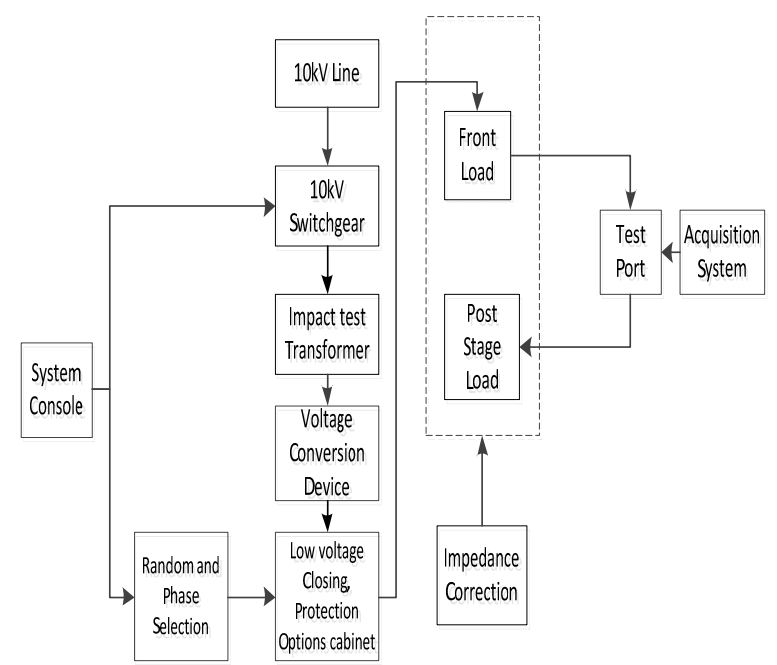

FIGURE I. BLOCK DIAGRAM OF LOW VOLTAGE CIRCUIT BREAKER SHORT CIRCUIT EXPERIMENT SYSTEM 


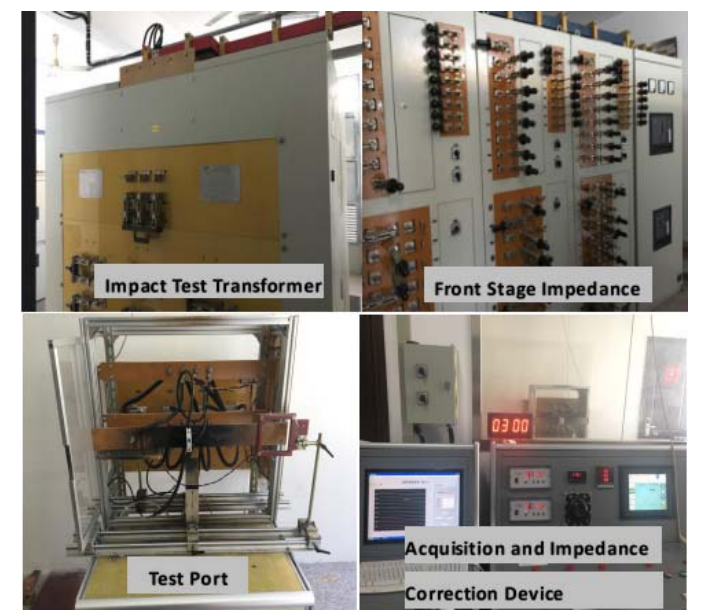

FIGURE II. DEVICE DIAGRAM OF LOW VOLTAGE CIRCUIT BREAKER SHORT CIRCUIT EXPERIMENT SYSTEM

From the test device of Figure II, it is known that there are a lot of connection terminals in the short circuit experiment system of the low voltage circuit breaker. In the process of the experiment, the oxidation phenomenon inevitably exists in the metal wire and the junction column. At the same time, after many large current experiments, the temperature rise of resistance, inductance and connection post will cause impedance and inductance change. All these factors will affect the test parameters of the short-circuit test. For the impedance correction of the short-circuit test system, the expected test parameters are input according to the needs of the test products. After calculating, we can get the impedance switch combination of the former and the later stages, and then set the impedance. After the short circuit test, read the expected TDMS data, get the actual input voltage, input current, power factor and other electrical parameters, judge whether the desired test parameters are reached. If the desired test parameters are met, if the result is satisfied, if the data is not satisfied, then the data is re calculated according to the size of the data deviation, and the new setting parameters are given. A short circuit test is conducted again to generate new expected waves until the requirements are met.

\section{Traditional METHOdS FOR SETting IMPEDANCE PARAMETERS OF SHORT CIRCUIT TEST SySTEM}

The traditional method of setting short circuit system test parameters and inquiring impedance switch data tables needs to be finished manually. The process is cumbersome and timeconsuming ${ }^{[5-6]}$. Taking the following experimental parameters as an example, the traditional setting method is introduced. The parameters are as follows: input $A C$ voltage $U=230 \mathrm{~V}$, output AC current $\mathrm{I}=7500 \mathrm{~A}$, power factor $=0.8$, traditional method of impedance parameter setting, as shown in Figure III. According to the above parameters, manual impedance and inductance data are manually calculated, and the resistance knife is manually inquired. Switch data meter, inductance knife switch data meter, according to the query data set impedance knife switch combination, will carry on a short circuit test, need to manually measure the actual output parameters to meet the expected parameters, if the data have deviation, repeat the above steps. Table I records the data collected each time. From the table data, it can be seen that the data gradually approximated the expected parameters. At the end of the seventh times, the impedance of R:0840, L:4210 and the switch data of the reactance knife were obtained, and the expected short-circuit current and power factor were obtained. But in the whole process, the number of repetitive operations is large and the efficiency is low. Therefore, it is urgent to improve the existing methods and reduce the number of operations.

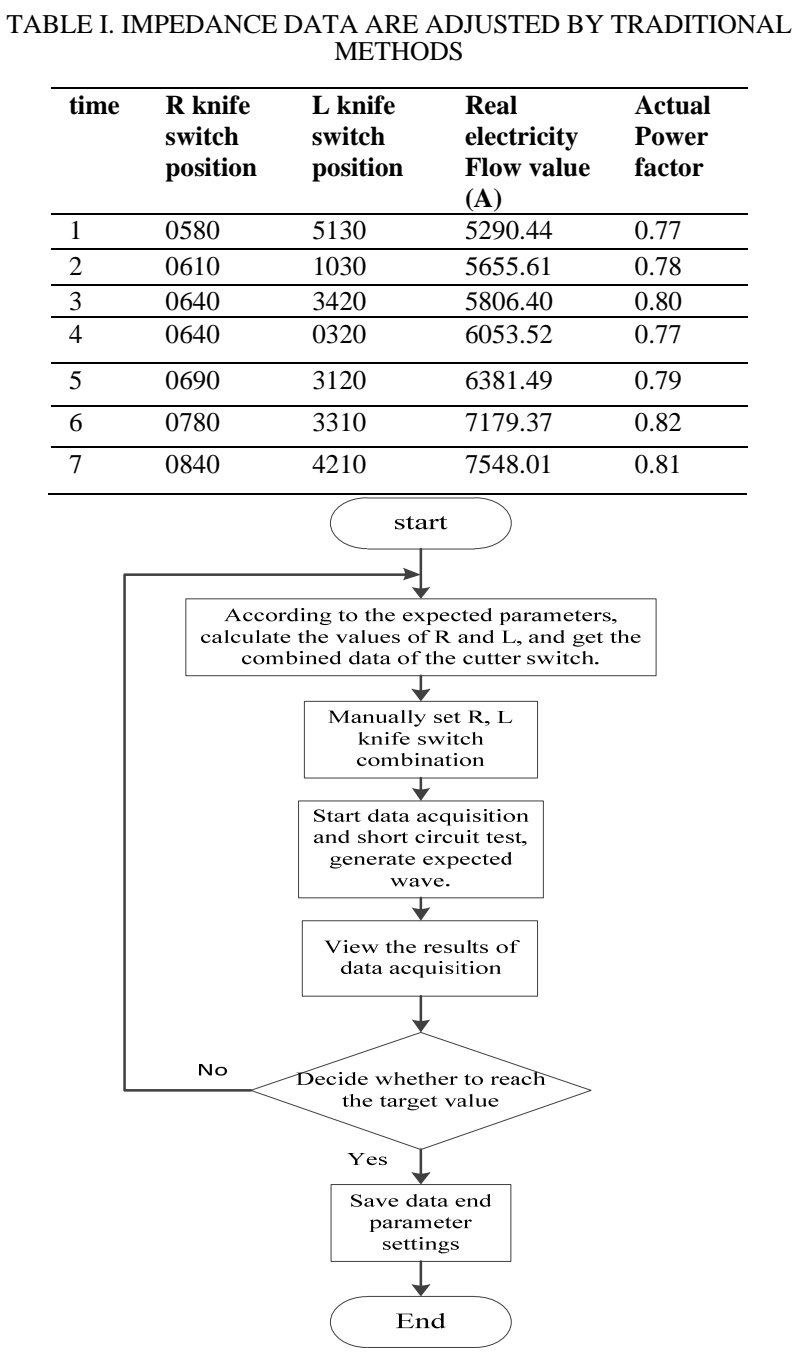

FIGURE III. USING TRADITIONAL METHOD TEST PARAMETER SETTING FLOW CHART

\section{ADAPTIVE IMPEDANCE CORRECTION METHOD FOR SHORT CirCUIT TEST SYSTEM OF LOW VOLTAGE CIRCUIT BREAKER}

In view of the disadvantages of traditional methods, the steps of parameter setting are improved, and the purpose of setting test parameters quickly is achieved through impedance correction. The concrete steps are as follows:

(1) Input the expected test parameters according to the test conditions of the products to be tested. 
(2) Automatic checking table calculates the resistance and inductance setting of the front and rear stages, and can adjust the resistance and inductance switch according to the position value.

(3) After the short circuit test, it automatically reads the expected wave TDMS data and calculates the actual parameter values to determine whether the expected test parameters are reached. If the desired results are met, the results are saved, otherwise, the error of the impedance is compensated according to the size of the data, and the new setting parameters are recalculated.

(4) Short-circuit test is performed to generate new expected waves, repeat steps 3 , until the requirements are met.

After the short circuit test, the size of the conduction current and the length of the conduction time will cause the resistance and inductance to be heated and the impedance change. In the software, the temperature changes of the terminals, resistance and inductance are measured by the multi point temperature measuring instrument, and the equivalent impedance of the circuit can be compensated to guarantee the more accurate ${ }^{[7-8]}$ of the data. The flow chart of the impedance correction software is shown in Figure IV. Figure V is the temperature change curve of the resistance, inductance and terminal at $20^{\circ} \mathrm{C}$ at room temperature, in which the current in Figure $\mathrm{A}$ is 6KA, and the current in Figure $\mathrm{B}$ is 10KA. As the contact resistance of the port terminal is larger and the temperature rise is obvious, the average temperature of the terminal terminal is up to $6.40^{\circ} \mathrm{C}$ with the $6000 \mathrm{~A}$ current as an example. The average temperature rises to $19.20^{\circ} \mathrm{C}$ under the current of the $10000 \mathrm{~A}$, so the impedance change caused by the temperature rise must be corrected.

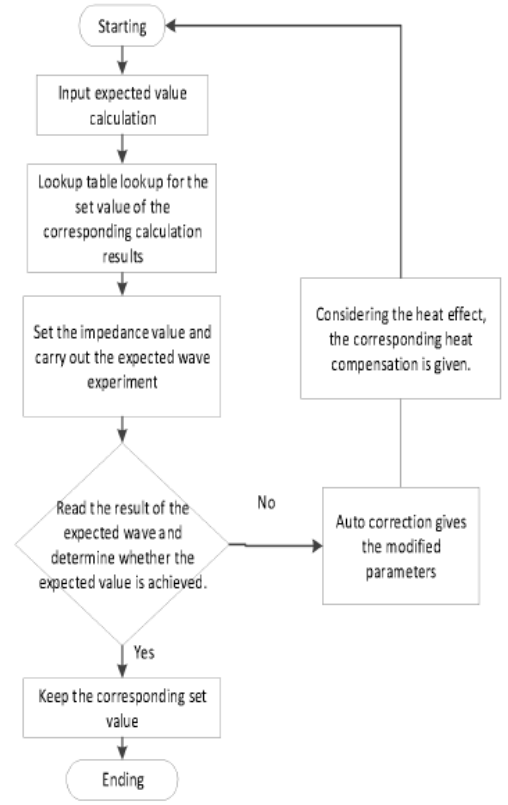

FIGURE IV. FLOW CHART OF IMPEDANCE ADAPTIVE CORRECTION METHOD
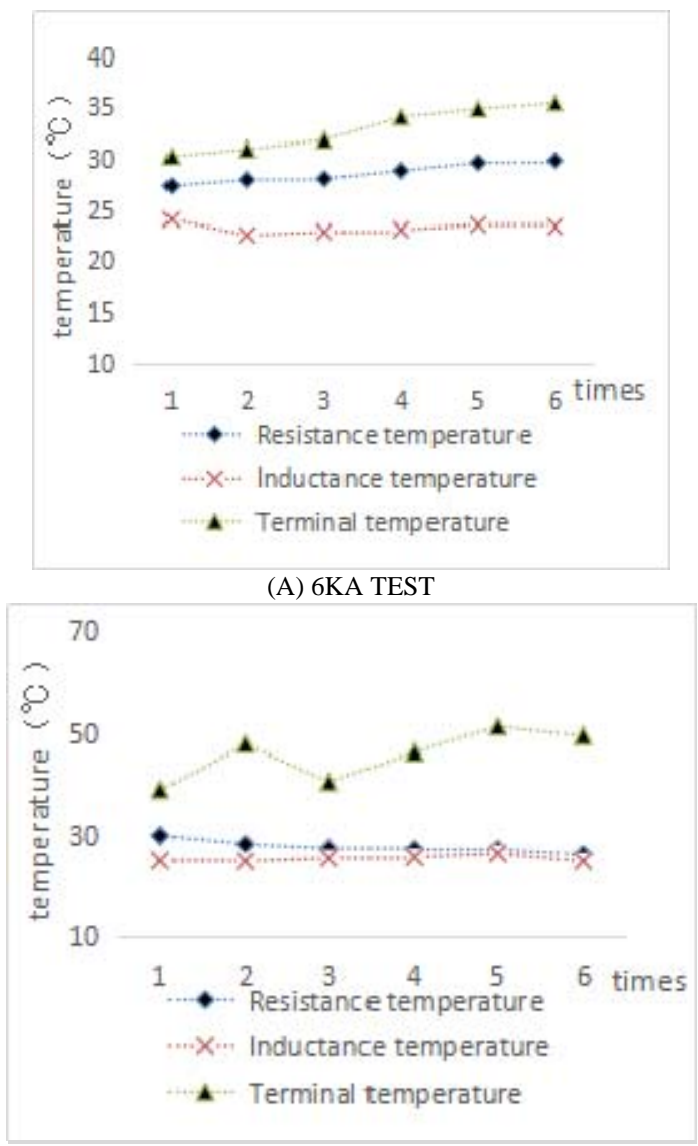

(B) 10KA TEST

FIGURE V. TEMPERATURE VARIATION CURVE OF IMPEDANCE AND INDUCTANCE CAUSED BY TEST

In this system, the simplified equivalent circuit diagram of the short circuit system is shown in Figure VI. The wiring column, wire and inductor in the system are all copper materials, and the resistivity temperature coefficient of copper is about 0.004/ degrees centigrade. At normal temperature range of 100 degrees Celsius, there is a linear relationship between resistivity and temperature.

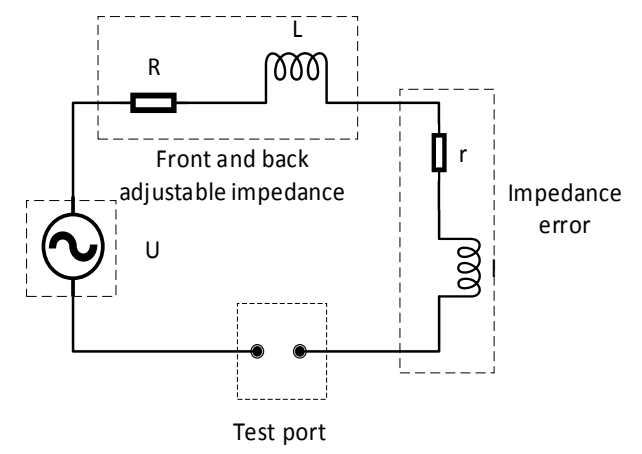

FIGURE VI. SIMPLIFIED DIAGRAM OF SYSTEM CIRCUIT

$\rho=\rho_{0}+(t-20) \times 0.004$

( $\Omega$ square millimetre / metre) 
The resistivity of copper at 20 degrees is 0.0172 , and the resistivity of aluminum at 20 degrees is 0.029 .

$$
R=\rho \frac{l}{S}=\left[\rho_{O}+(t-20) \times 0.004\right] \frac{l}{S} \Omega
$$

Taking 10 square millimeter and 50 centimeter copper wire as an example, the resistance changes at 20 degrees centigrade.

$$
r=[(40-20) \times 0.004] \times 0.5 / 10=0.004 \Omega
$$

Under the condition of large input current, the change of resistance of 4 milliohms will affect the test results. It is necessary to correct the parameters in order to obtain accurate parameters.

As an example of setting the test parameters in front: $\mathrm{I}=7500 \mathrm{~A}, \mathrm{U}=230 \mathrm{~V}$, power factor $=0.8$, compared with the traditional method, after calculation and experiment, the parameters are corrected until the expected parameters are met. The repeated cycle times of the impedance adaptive correction method for the short circuit test system of the low voltage circuit breaker are 2 times, and the requirements of the test parameters can be reached. Through software correction, the number of impedance adjustment times can be reduced from 7 times in the original table to 2 in Table II, and the parameters such as short circuit current and power factors are accurately obtained.

\section{TABLE II. EXPERIMENTAL DATA OBTAINED BY IMPEDANCE} CORRECTION METHOD

\begin{tabular}{lllll}
\hline time & $\begin{array}{l}\text { R knife } \\
\text { switch } \\
\text { position }\end{array}$ & $\begin{array}{l}\text { L knife } \\
\text { switch } \\
\text { position }\end{array}$ & $\begin{array}{l}\text { Real } \\
\text { Current } \\
\text { value (A) }\end{array}$ & $\begin{array}{l}\text { Actual } \\
\text { power } \\
\text { factor }\end{array}$ \\
\hline 1 & 0790 & 3130 & 7290.63 & 0.76 \\
\hline 2 & 0840 & 3420 & 7506.40 & 0.80 \\
\hline
\end{tabular}

\section{IMPLEMENTATION OF IMPEDANCE ADAPTIVE} CORRECTION SOFTWARE

\section{A. Software Design Process}

In the whole software, the user needs to enter the expected parameters, such as the expected voltage, the expected current, the expected power factor, and then click the calculation button to get the expected resistance and expected inductance data. The user clicks on the query button, chooses the required files to enter the query, and gets the combination value of the knife switch. After setting the resistance and inductance knife switch, the expected wave is obtained, and the TDMS file is saved. The TDMS file button is read by click and read, and the required TDMS files are selected to read the data. The effective voltage, the effective current, the peak voltage and the peak current are displayed, and the waveform of the voltage and current is displayed, and the user clicks on the calculation button. The effective inductance and effective resistance are obtained. The user reads the voltage from the system console in the system console, inputs and corrections the impedance. Finally, the corrected resistance and inductance are obtained. Using the query button, the more accurate combination of the knife switch can be obtained. The specific flowchart is shown in Figure VII, and the TDMS data read flow chart is shown in Figure VIII.

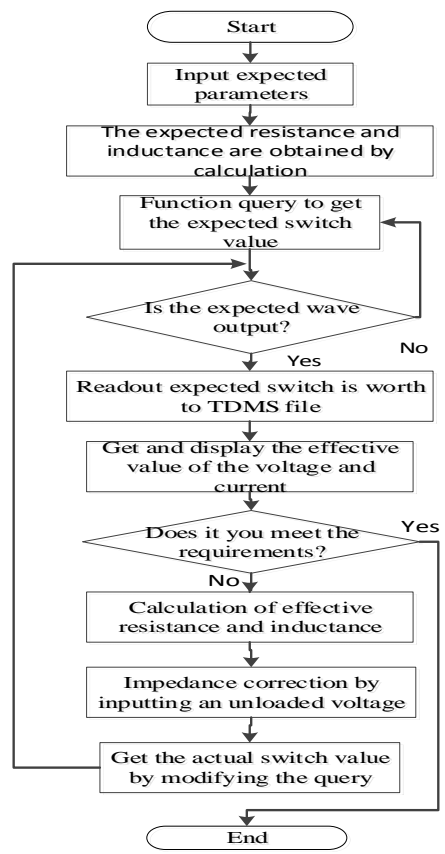

FIGURE VII. SOFTWARE FLOW CHART OF SYSTEM IMPEDANCE CALCULATION AND CORRECTION

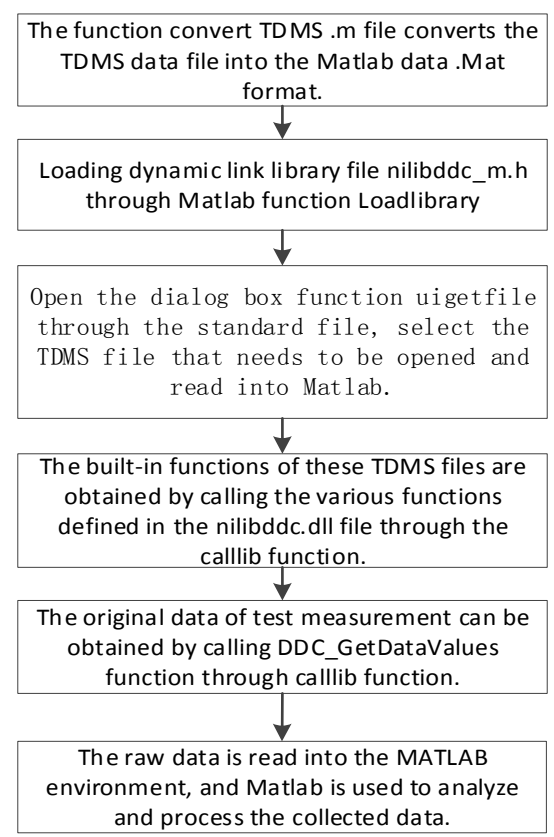

FIGURE VIII. TDMS DATA READING FLOW CHART ${ }^{[9-10]}$

\section{B. Parameter Calculation and Design of Impedance Query} Function

The user enters the expected line voltage $U a b=380 \mathrm{~V}$, $\mathrm{Ubc}=380 \mathrm{~V}$, Uca $=380 \mathrm{~V}$, the expected current $\mathrm{I}=1000 \mathrm{~A}$, the expected power factor beta $=0.8$, click the calculation button, 
and the result, as shown in Figure IX, obtains the three phase resistance and inductance data. Click the resistance query and the inductance query button, select the corresponding impedance and the inductive switch combination file to get the switch value of the three phase resistance and inductance, as shown in Figure X.

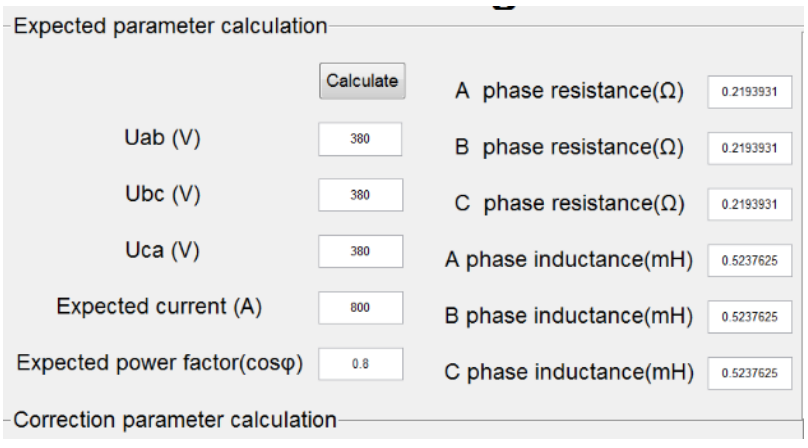

FIGURE IX. FUNCTION DESIGN FOR EXPECTED PARAMETERCALCULATION

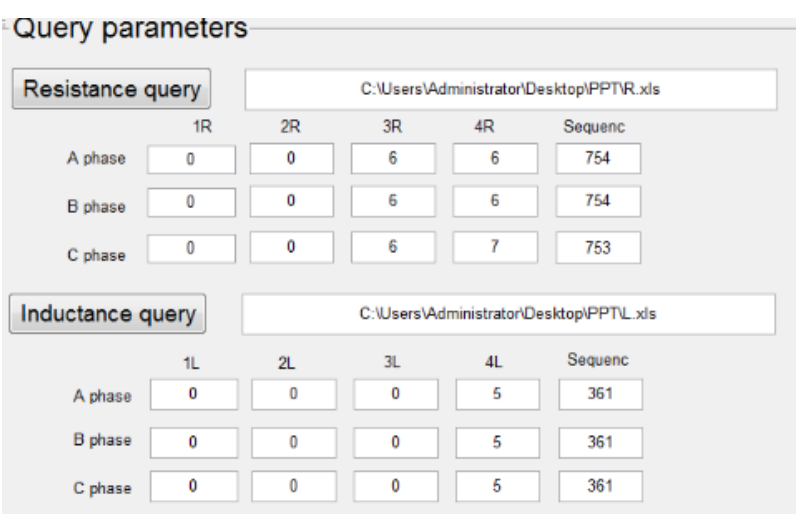

FIGURE X. FUNCTION DESIGN OF QUERY PARAMETERS

\section{Modified Parameter Calculation and Query Function Design}

Click and read the TDMS file, calculate the actual voltage and inductance value, the power factor, input the parameters, click the correction button, get the corrected resistance and inductance, and display the voltage and current waveform, as shown in Figure XI. The function design of the modified parameter is shown in Figure XII, which is consistent withthe TDMS filed ata.

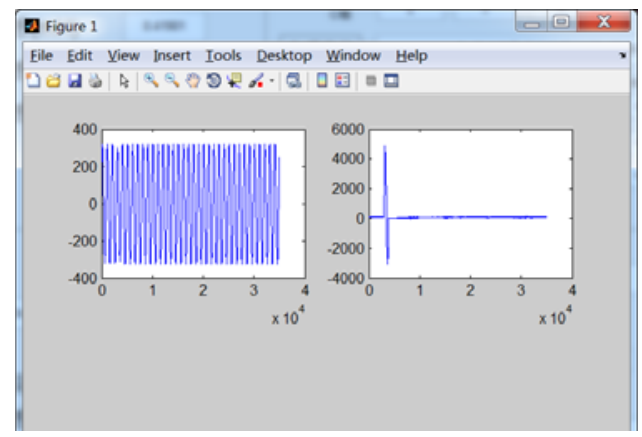

FIGURE XI. FUNCTION DESIGN OF VOLTAGE AND CURRENT WAVEFORM DISPLAY

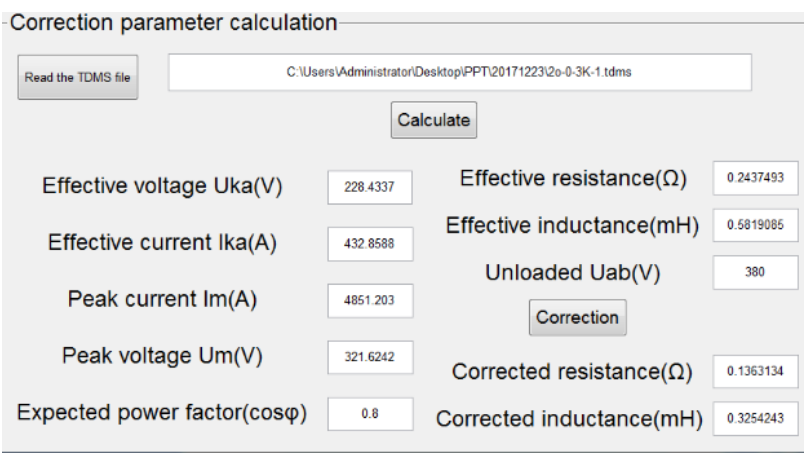

FIGURE XII. FUNCTION DESIGN FOR MODIFIED PARAMETER CALCULATION

The TDMS file parameters are calculated by selecting the discrete data of a $\mathrm{N}$ point in a cycle to calculate the true value of the voltage and current, such as (4) and (5).

$$
\begin{aligned}
& U_{r m s}=\sqrt{\frac{1}{N}\left(\sum_{k=0}^{N-1} u_{k}^{2}\right)} \\
& I_{r m s}=\sqrt{\frac{1}{N}\left(\sum_{k=0}^{N-1} i_{k}^{2}\right)}
\end{aligned}
$$

The calculation formulas of active power and power factor, such as (6), (7):

$$
\mathrm{p}=\frac{1}{N} \sum_{k=1}^{n} u_{k} i_{k}
$$

$$
\cos \varphi=\frac{P}{U_{r m s} I_{r m s}}
$$

Among them, UK and IK represent equal interval and discrete voltage and current values in TDMS data table.

The corrected resistance and inductance data obtained from the plate can be calculated and inquired from the corrected calculation parameters, and the corresponding resistance and the inductor switch data sheet file are selected to get the required switch value, as shown in Figure XIII. 


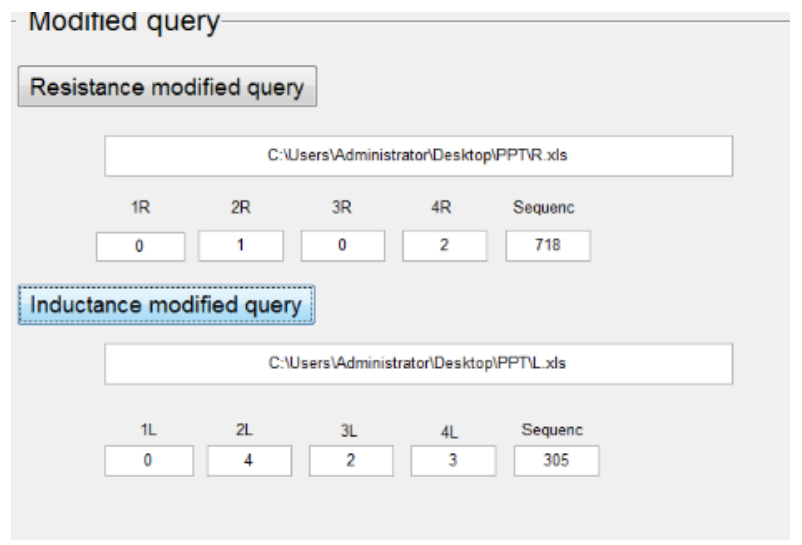

FIGURE XIII. MODIFIED QUERY FUNCTION DESIGN

\section{Interface Design of Adaptive Correction Platform for Short Circuit System Impedance}

The expected parameters are calculated with the plate, the query parameter plate, the corrected parameter calculation plate and the correction query plate interface to make the internal connection of the plates. The software interface of the final debugging operation is shown in Figure XIV, and the software can quickly realize the function of parameter technology, impedance correction, waveform display and so on.

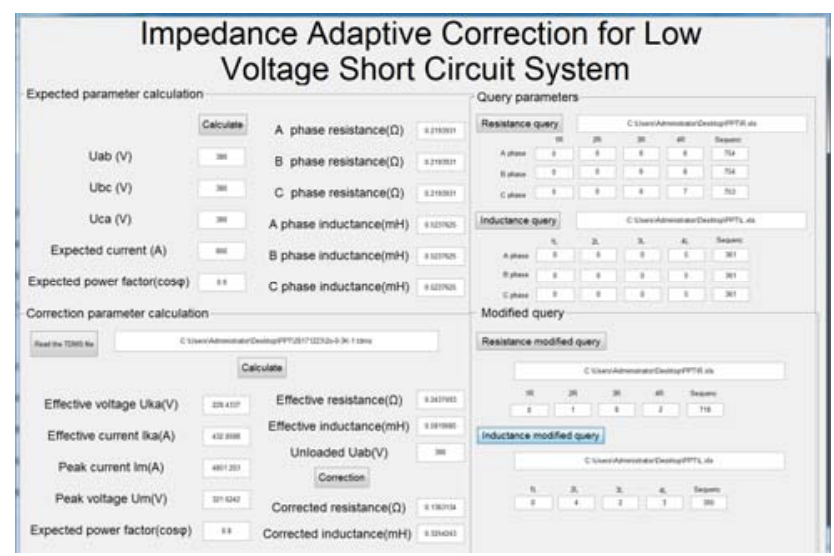

FIGURE XIV. OVERALL DESIGN OF AN IMPEDANCE ADAPTIVE CORRECTION PLATFORM

\section{CONCLUSION}

In the impedance adaptive correction method introduced in this paper, the user can realize the parameter calculation only by input parameters, can obtain the required impedance switch value through the query interface, start the system to output the expected wave and generate the TDMS file, the correction software can read the TDMS file, and display the waveform diagram of the actual voltage and current, and the user can Impedance correction is achieved and accurate impedance switch values are obtained. This method mainly has the following advantages: software calculation test parameters instead of manual calculation, automatic lookup table instead of manual lookup, improve efficiency, automatically read the expected wave TDMS file, and measure the actual electrical parameter data, and consider the factors such as the impedance temperature effect, the contact resistance oxidation of the switch knife gate, the circuit impedance and so on. The error is adjusted adaptively to ensure the accuracy of the test parameters and greatly improve the test efficiency.

\section{ACKNOWLEDGEMENTS}

This article is funded by LQ16E070004, the Natural Science Foundation of Zhejiang Province, 2016C31052,the Public Technology Research Project of Zhejiang Province, Y201737046,the Education Department Project of Zhejiang Province and G20150006,the Science and Technology Bureau project of Wenzhou City.

\section{REFERENCES}

[1] Li He, Jiang Ning, Feng Jianqiang. Study on closed circuit in short circuit test of low voltage circuit breaker [J]. electrical and energy efficiency management technology, 2017 (10): 52-55.

[2] Li Xingwen. Summary of new R \&amp; D technologies for low voltage circuit breakers [J]. electrical appliances and energy efficiency management technology, 2015 (09): 1-7.

[3] Jin Xuefeng, Chen Yunan, Tong Xiang. Short circuit test analysis of DC traction power supply system [J]. urban rapid rail transit, 2016,29 (03): 103-105.

[4] Zhang Hao, Xie Qiuhui, Cai Chunhao, Fu Peigang, Zhao Yong, Zheng Lun. A new overcurrent detection scheme for low voltage circuit breakers [J]. electrical and mechanical engineering, 2015,32 (02): 285289.

[5] Zhang Hao, Xie Qiuhui, Cai Chunhao, Fu Peigang, Zhao Yong, Zhu Minglei, Ju Hong Quan, Wu Fei, Zheng wheel. A new type of low voltage circuit breaker large current test equipment $[\mathrm{J}]$. mechanical and electrical engineering, 2016,33 (04): 476-482.

[6] Liu Yi. Effect of short circuit test parameters on circuit breaker test [J]. low voltage electrical appliances, 2009 (09): 12-15.

[7] Wang Feng, Zhao Yi, jungle. Study onthermal stability during short circuit test [J]. electrical and energy efficiency management technology, 2017 (17): 56-60.

[8] Tao Ye, Wang Jiwei, Pei Liying. Analysis of voltage disturbance caused by short circuit test of circuit breakers [J]. power supply, 2017,34 (06): 82-84.

[9] Feng Weiwei, Yang Shiwen, South Jin Rui, Ma Yucheng. LabVIEW TDMS file and SQL database data processing speed research [J]. instrument and analysis monitoring, 2011 (01): 10-12.

[10] Yujie Lin, Xiangyuan Bu, Shuai Wang, Yuan Chai, Jianping An. Impact of Phase Noise on TDMS Based Calibration for Spaceborne Multi-Beam Antennas [J].China Communications,2018,15(03):128-136. 